\title{
PENSAR EL ORDEN Y EL DESORDEN: UNA POÉTICA DE LA EXCEPCIÓN
}

Daniel Innerarity

Universidad de Zaragoza

«El espíritu va, en su trabajo, de su desorden a su orden. Es importante que conserve hasta el final los recursos del desorden y que el orden que ha comenzado a darse no le atrape tanto, ni se le convierta en un maestro tan rígido, que no pueda cambiarlo y utilizar su libertad inicial» (Paul Valéry 1960, 714).

Vivimos en un tiempo en el que nada se conquista con absoluta seguridad, ni el saber ni la competencia. La novedad, lo efímero, la sucesión rápida de informaciones, de productos, de modelos de comportamiento, la necesidad de efectuar frecuentes adaptaciones, la exigencia de flexibilidad, dan la impresión de que vivimos únicamente en el presente y de un modo que impide toda estabilización. Inscribir algo en la duración parece algo de menor importancia que valorar el instante y el acontecimiento. Ahora bien, el pensamiento siempre ha tenido algo que ver con las operaciones de ordenar y clasificar, con la pretensión de otorgar un sentido estable a la variedad desordenada de las manifestaciones de la realidad. Esta articulación de lo disperso, si puede seguir teniendo algún sentido, ha de hacerse cargo de las paradojas del orden y la organización. Y así ha venido ocurriendo últimamente: desde la ciencia hasta la teoría de las organizaciones, en el nivel de los conceptos y en los modelos para la acción, la conciencia del desorden y la irregularidad se intensifica. Se trata de una dificultad tanto teórica como práctica, que obliga a pensar nuevamente el desorden en todas sus manifestaciones, como desorganización, turbulencia, caos, complejidad o entropía.

Los nuevos escenarios del pensamiento están diseñados para hacerse cargo de dinámicas no lineales, estructuras disipativas, orden por fluctuación, desequilibrio habitual, sistemas complejos y abiertos, irrupción de lo nuevo y esta- 
bilizaciones relativas. Pensar es una tarea que exige haber caído en la cuenta de que el orden se esconde en el desorden, lo aleatorio actúa constantemente, que la consideración del movimiento y sus fluctuaciones resulta más significativa que las estructuras y las permanencias. De ahí que, frente a la concepción maximalista del orden, contra la taxonomía definitiva en virtud de la cual las cosas encontrarían una disposición estable en una totalidad armónica, resulta necesario elaborar algo así como una epistemología poética de la excepción, a partir de la experiencia de los inconvenientes del orden para la vida, del rendimiento cognoscitivo del desorden y la excepción, y de los límites de toda clasificación.

\section{El saber desordenado}

La más célebre constatación del desorden del saber procede de la imaginación de Borges. Está, por un lado, el texto tantas veces citado en el que se refiere la extraña clasificación de los animales en cierta enciclopedia china, texto que constituirá el punto de partida de Las palabras y las cosas de Foucault (1993). Allí los animales se encontraban divididos en a) pertenecientes al emperador, b) embalsamados, c) amaestrados, d) lechones, e) sirenas, f) fabulosos, g) perros sueltos, h) incluidos en esta clasificación, i) que se agitan como locos, j) innumerables, k) dibujados con un pincel finísimo de pelo de cameIlo, I) etcétera, m) que acaban de romper un jarrón, n) que de lejos parecen moscas (Borges 2003a). Están también otros relatos suyos acerca del carácter imposible de las bibliotecas entendidas como memorias exactas de la humanidad o representaciones fieles de lo que se sabe. En El congreso, por ejemplo, se nos cuenta el esfuerzo inútil de unos individuos de América latina que deciden crear un congreso mundial y una biblioteca adjunta, y que no terminan de ponerse de acuerdo acerca de su composición. Enormes paquetes de libros se amontonan sin catalogar en una cueva. Finalmente deciden pegarles fuego y abandonar el proyecto tras haberse dado cuenta de que englobaba al universo entero (Borges 2003b). La realidad y su representación están enemistadas por un hiato imposible de salvar.

Es posible que esta intuición de Borges sea el origen de muchos relatos que han hecho de la clasificación del saber una tarea paradójica, absurda e imposible. Entre las clasificaciones fantásticas propuestas a los bibliotecarios en una época postmoderna cabe destacar de Paul Braffort, en Les Bibliotheques invisibles, donde propone ordenar los libros a partir de sus títulos literales y en torno a criterios como los colores (criterio que serviría para clasificar libros como El perro amarillo de Simenon, o Las flores azules de Queneau), el calendario (que agruparía títulos como Martes de Melville, El Jueves Santo de Bossuet, El 18 de Brumario de Marx, El otoño de la Edad Media de Huizinga) o familiares (donde se encontrarían, por ejemplo, Los hermanos Karamazov de Dovstoievski, La cabaña del tío Tom de Harriet Stowe, El sobrino de Wittgenstein de Bernhard). Otro criterio, más exacto, pero tan inservible a efectos de clasificación y organización del conocimiento se encuentra en la obra Invitado a una decapitación, de Vladimir Nabokov, donde el bibliotecario de una prisión llevaba un 
catálogo que clasificaba las obras según el número de páginas. Estos y otros relatos similares surgen de una misma experiencia cultural: al subrayar la arbitrariedad del orden en lo que tiene de inútil o ridículo, el saber es pensado como algo que no puede organizarse con sentido, como algo monstruoso.

La literatura registra así un problema que trasluce algunas propiedades del saber en el mundo actual; muestra la comicidad de una situación en la que se encuentran los seres humanos en las Ilamadas sociedades del conocimiento. Estas historias apenas tendrían sentido en un universo más limitado, sin el volumen de conocimiento que nos vemos obligados a gestionar y las enormes dificultades que plantea. Y es que las bibliotecas y los archivos no son meros lugares en los que se almacenan libros y documentos, sino sobre todo sistemas de clasificación y ordenaciones elaborados de acuerdo con una lógica que evoluciona con el paso del tiempo pero que siempre ha pretendido hacer del saber algo disponible. Los sistemas de ordenación constituyen un sistema de representación del saber, como por ejemplo el árbol de Porfirio que ha tenido una larga vida hasta Diderot, mientras ha sido capaz de reflejar la complejidad de los saberes y su articulación. Actualmente hay otros modelos como la red, el mind map o el rizoma que parecen haber superado al anterior, inservible por exceso de jerarquización y simplicidad. Estos modelos intentan responder al problema de cómo pensar el orden y la articulación de los saberes en un escenario más complejo, que no puede manejarse con la tradicional sistemática bibliotecaria. En internet, ninguna máquina de búsqueda necesita por ejemplo una jerarquización de los conceptos; la articulación de temas y contenidos escapa de cualquier metaestructura lógica, sin caer por ello en el caos o la completa inabarcabilidad. El saber parece flotar libremente, más allá de títulos y rúbricas. Su incrementada accesibilidad parece estar en conexión con la pérdida de significación de las posibles estructuraciones.

Estas y otras dificultades semejantes invitan a pensar de nuevo el orden del conocimiento sin desentenderse cómodamente de las paradojas que plantea cualquier clasificación. Y probablemente exigirá que nos despidamos de la idea de un orden cultural en el que cada cosa tiene su sitio, un orden trascendental e incuestionado. El saber, como el orden social, es siempre algo inestable, desprotegido y hostigado, cualquier cosa menos una conquista imperturbable, protegida frente a toda desestabilización. También en el orden del saber la tranquilidad es siempre engañosa, una tregua con fecha de caducidad. La estabilidad ha merecido desde antaño la desconfianza y la sospecha, incluso la declaración de imposibilidad. Al mismo tiempo, una cierta cantidad de orden parece necesaria para arreglárselas con la realidad y no resulta posible actuar sin suponer una constancia, aunque sea mínima, en las condiciones del mundo. Ante estas exigencias contrapuestas y a la vista del incremento de la complejidad que plantea una sociedad del conocimiento, ¿cabe todavía hablar, y en qué condiciones, de regularidad, orden y clasificación? 


\section{La inexactitud de las reglas}

Toda la cuestión del orden, su complejidad y posibilidad, se juega en el esclarecimiento de qué significa la operación de seguir una regla. Sobre esta cuestión del rule following hay ya toda una vieja discusión que, en la filosofía más reciente, ha generado una serie de conceptos que pretenden incomodar un tanto la distinción simple entre el orden y el desorden, entre la conducta de seguir una regla y vulnerarla, entre lo prohibido y lo obligatorio. Pensadores como Luhmann (1964), Waldenfels (1987), Elster (1989), y Bourdieu (1987) coinciden en hablar de una zona ambigua, un umbral, un espacio de juego y maniobra, de in-diferencia en relación con el par regla/excepción.

Pero el asunto tiene ya sus orígenes en Kant, tal vez el primero en reconocer la inevitable inexactitud de las reglas que orientan las destrezas humanas. Su formulación se centra en el problema de la transposición de la teoría al ámbito de la praxis, lo que parece simbolizar el núcleo en el que se contiene alguna inexactitud más general de la vida humana. Kant vio que la idea de prescribir la aplicación de la regla en la regla misma conduciría hacia un regreso al infinito. En el escrito Sobre el dicho común: esto puede ser verdad para la teoría pero no para la praxis (1908, VIII, 275) rechaza esa pretensión de que el paso de la teoría a la praxis pueda ser regulado con absoluta precisión; no hay reglas para determinar si algo es el caso de una regla, no es posible que las reglas establezcan inequívocamente cuándo y cómo deben aplicarse, para lo que se requiere una capacidad de juicio específica; la aplicación exige siempre interpretación y decisión, lo cual implica una cierta inexactitud similar a la intuición artística, al ingenio o la sutileza de que hablará en la Antropología (1908, VIII, § 44) como una capacidad respecto de lo concreto. Se trata de algo que propiamente no puede enseñarse ya que la enseñanza tiene lugar siempre a través de reglas.

El otro gran hito de esta reflexión es el conocido análisis de Wittgenstein sobre la conducta de seguir una regla. Según él, aunque en ocasiones existen reglas de aplicación de reglas (1958, 90; J. Vicente Arregui 1988), es decir, aunque a veces se pueden dar reglas de segundo orden que regulan la aplicación de otras reglas de primer orden, el proceso de justificación de una acción por referencia a unas reglas, alcanza un término. Llega un momento en tal proceso de justificación en el que no se puede ya invocar reglas ulteriores, y queda sólo la acción. La cadena de razones que podemos esgrimir para justificar que seguimos una regla tal como lo hacemos, tiene un límite. Al final de la serie de razones, o al final de la cadena de reglas que regulan cómo se han de aplicar las reglas, hay una espontaneidad de la acción. Una regla, por grande que sea el número de veces que ha sido aplicada en el pasado, no determina en el presente ningún modo de actuar.

Esta inexactitud de las reglas se debe fundamentalmente a su escaso poder de hacerse cargo del contexto. Las reglas pueden especificar contextos pero esa determinación es siempre incompleta porque, en primer lugar, los contextos se solapan y entrecruzan y, por otro lado, los contextos de aplicación no se pueden acotar completamente. Buena parte de los errores que cometemos se 
debe a una equivocada identificación de contexto (Bateson 1983, 374). Se equivocaría de contexto, por ejemplo, un espectador en el teatro que al escuchar a Hamlet hablando a Ophelia de suicidio se le ocurriera Ilamar a la policía o al médico. Ordenar el poemario de Sánchez Ostiz que Ileva por título El santo al cielo entre los libros de religión es un típico fallo de bibliotecario que se atiene a la literalidad de las palabras sin observar el contexto en el que se inscriben. Por una razón semejante resultan inservibles las máquinas de traducir automáticamente. Hacerse cargo del contexto es la operación más propia de la inteligencia, aquella que no puede ser sustituida por un mecanismo o una regla exacta.

Y es que la verdad que pretendemos, como el bien o la justicia, no son asuntos de precisión matemática sino que están inscritos en un contexto vital sin el que resultan ininteligibles. El contexto confiere a los asuntos humanos una significación más rica y más compleja que la exactitud de los automatismos. Hay cosas verdaderas pero inoportunas; otras eran verdaderas y ya no lo son; algunas son verdaderas y nadie lo sabe; además de lo verdadero existe lo relevante, lo significativo, lo interesante... La parcialidad e inevitabilidad de los contextos se debe a la inserción de las cosas en ámbitos de sentido a los que no llega ninguna regla exacta. Es la imprecisión misma de la vida, por la que tenemos continuamente que elegir, interpretar y aplicar las normas a una determinada situación. Pero la relación entre la regla y la aplicación está sujeta a unas paradojas -advertidas en la tradición filosófica que va desde Kant a Derrida- en virtud de las cuales la aplicación no sólo cumple las reglas sino que las complementa, modifica y suspende. Existe algo así como un auto-deconstrucción de las reglas que corresponde a lo que Derrida ha llamado différance: la vulneración de las normas es condición de posibilidad de su aplicación, lo que también posibilita la libertad de encontrar lo nuevo. Seguir una regla implica siempre la selección entre una variedad de reglas y, por tanto, una decisión acerca de cuál entre ellas es la relevante, decisión que no está asegurada por esas mismas reglas, y de modo que el cumplimiento de una regla supone con frecuencia la vulneración de otras.

Toda aplicación de reglas tiene algo de lesión de esas reglas. Está la vieja idea de que las excepciones confirman la regla; la excepción justificada por el bien de la causa; la descortesía de la puntualidad y el retraso acordado, cum tempore; la epiqueia en la teología moral; la discreción que se permite a la hora de aplicar normas y reglamentos... ¿Por qué la excepción confirma la regla vulnerándola? Pues porque el sentido de las reglas no es pretender una validez sin excepción, porque la excepción no tiene su sede fuera sino dentro de la regla. De alguna manera, la regla tiene que prever su excepción en orden a su propia elasticidad y fortaleza.

La idea de una regresión hasta el infinito vuelve a plantearse, en la práctica, cuando un sistema tiene que hacer algo para regular las excepciones y muchas instituciones tienen indicaciones en este sentido. En esos casos, lo que se plantea es la posibilidad de aprender a tramitar lo inusual, es decir, de extraer un último resto de regularidad en los casos irregulares, de crear algo así como una 
rutina con lo excepcional: por ejemplo qué hacer ante una catástrofe o cómo regular el estado de excepción. Ahora bien, regular lo que ha de hacerse en caso de excepción es algo paradójico ya que tiende a hacer de la excepción el caso normal, a normalizarla: proporcionar una regla para todas las excepciones, que para esa regla no serían una excepción. Pero cualquier regla genera una excepción. Y esa excepción no puede regularse porque lo excepcional, en tanto que imprevisto, no es plenamente anticipable. A pesar de lo cual, en la práctica se pueden proporcionar algunas reglas explícitas para casos de excepción. Tal es el objetivo de las "patterned evasions»: el establecimiento de normas que regulan la vulneración de normas. Su inevitable paradoja se hace visible en el caso especial de las falsas alarmas. Cuando las alarmas son demasiado frecuentes, terminan por no ser atendidas de manera regular. Se trata de una rutina que es fatal en el caso en que la alarma resulta no ser falsa. El hundimiento del Titanic es uno de los casos más célebres de esa despreocupación rutinaria pese a la insistencia de la alarma. Determinar cuándo estamos ante un «caso excepcional» es algo que necesariamente queda en alguna medida inespecificado, que requiere ese juicio del que hablaba Kant o, por decirlo con Gadamer, sensus communis (1990, 36).

Si la aplicación de reglas resulta tan imprecisa, bien cabe hablar de una poética de la excepción. La aplicación de reglas es una conducta estética en la medida en que ninguna regla contiene en sí misma el método de su aplicación. Si una ley contuviera en sí misma el método de su aplicación, enton-ces no habría ningún libre juego entre la acción y la ley, y la conducta de seguir una norma sería un puro automatismo mecánico que no dejaría lugar para la libertad en ningún sentido relevante. En cambio, nos resulta algo bastante natural y evidente que haya algo así como vulneraciones de las reglas, por ejemplo, en el lenguaje, cuyo potencial no se puede reducir a un conjunto de reglas o procedimientos. Así lo recuerda la poesía o el procedimiento metafórico. Similarmente los juristas hablan de «interpretación constructiva», lo que testifica que la interpretación es siempre creativa. El momento heurístico de la razón indica que hay un cierto saber involucrado en cualquier aplicación de una ley, reglamento u orden, que la conducta de seguir una regla está mediada por la interpretación de la norma y supone una habilidad específica que estriba precisamente en saber utilizarla (J. Vicente Arregui 1988). El hecho de que ninguna regla contenga en sí misma el método de su aplicación significa que en la conducta de seguir una regla está implicado siempre un cierto tipo de conocimiento, una capacidad inventiva que cabe explicar por analogía con los procedimientos de la imaginación poética. Al final va a resultar que sin imaginación no hay conducta buena ni orden razonable, que el bien y la verdad tienen más que ver con la estética de lo que pensábamos.

\section{La repetición imposible}

Una regla es un procedimiento general que implica una cierta repetición. La prosecución o aplicación de reglas es una experiencia cotidiana de cualquiera, desde la praxis de un bibliotecario hasta la decisión de un juez. En la orga- 
nización de los saberes, en la formación de la conciencia y en el aprendizaje, en las sociedades y las organizaciones, la repetición juega un papel muy importante. Schütz hablaba de la utilidad antropológica del «etcétera», sin lo que seríamos incapaces de realizar cualquier movimiento (1971, 153). Las reglas y la normas son irrenunciables para la estabilización institucional, porque en la vida es necesario el saber a qué atenerse, la expectativa de las repeticiones. "Hacer lo mismo bajo las mismas circunstancias» significa repetir; lo que las organizaciones organizan en principio es la repetición; las reglas son procedimientos repetibles.

Esta principio de repetición no deja de ser aporético. Seguir una regla significa actuar del mismo modo bajo las mismas circunstancias. El uso de la palabra 'regla' está unido al uso de la palabra 'igual' (Wittgenstein 1984, § 225). Pero ni las circunstancias ni los modos de actuar pueden ser nunca exactamente los mismos. Con lo cual hemos de añadir: «igual desde un punto de vista relevante»o «igual en lo esencial», sin poder indicar qué significa aquí relevante o esencial. De este modo surgen zonas de indeterminación: Ios criterios de similitud, proporción, relevancia sólo se pueden obtener en un contexto práctico que no se pueden articular nunca definitivamente como un complejo de reglas. La praxis sobrepasa, excede y deconstruye las prescripciones. Wittgenstein, Bourdieu o Derrida lo han mostrado en el concepto de juego. En su intento de hacer relevantes para las ciencias sociales algunos aspectos de la filosofía de Wittgenstein, Peter Winch (1990) afirmaba que sólo podemos saber si dos cosas han de ser tratadas de igual modo si se nos comunica el contexto en el que se plantea esa cuestión.

Aquí hay una paradoja que hace de la repetición algo imposible. Kierkegaard, Deleuze o Derrida han Ilamado la atención sobre este hecho al considerar que la repetición, la acción sometida a reglas, es falsificadora: todo aseguramiento institucional implica fragilidad, toda aplicación de reglas o imitación supone una creación singular, toda legislación es posterior. Kierkegaard lo sentenció así: la dialéctica de la repetición es sencilla, pues lo que se repite ha sido; si no, no podría repetirse. Pero precisamente esto, que haya sido, convierte a la repetición en algo nuevo $(1991,212)$. Freud vino a decir algo parecido: en la repetición se fija algo que no puede ser fijado (1982, 245). El gozo de los niños por escuchar la misma historia o repetir el mismo juego procede de que no han hecho la experiencia de la caducidad, de lo irrecuperable; en ellos las repeticiones todavía son puras. La madurez parece ser una especie de conciencia de lo irrepetible, de que, por decirlo con una expresión de Lacan, la repetición es un encuentro fallido, algo parecido a una cita perdida.

Si toda repetición -toda regularidad- resulta inevitablemente fallida, por pequeña que esa anomalía pueda parecer, esto significa que en toda aparente repetición puede descubrirse algo singular. En cibernética y teoría de sistemas hay un concepto para explicar este tipo de cosas: recursividad. Algo es puesto, luego aplicado, pero esa aplicación es reintroducida en el proceso de constitución. Existe algo así como una insistencia de lo particular, del caso particular, una resistencia indiosincrática, que convierte a toda ciencia y a toda pra- 
xis en una tarea interpretativa. Hay un momento de interpretación que limita la subsunción y relativiza las generalizaciones, recontextualiza. Refiriéndose al caso concreto del derecho, Derrida habla de que todo caso es otro y necesita una interpretación completamente distinta, que no puede ni debe ser sustituida por ninguna regla existente, registrada o codificada (Derrida 1994). En otro caso, estaríamos ante una operación mecánica. Desde Heráclito al menos hay una cierta coincidencia en asegurar, hablando con propiedad, qué no puede ser una repetición: reproducción idéntica. La repetición nunca es pura, Ileva en sí la marca de una diferencia constitutiva. Tiene que haber un suplemento, algo adicional, ulterior, dado que la aplicación de reglas nunca es repetición en el sentido de replicación asegurada, mera reproducción.

\section{Gestionar la excepción}

La experiencia primordial que arrojan los temas hasta ahora mencionados es la conciencia de la finitud del orden y una radicalización de la idea de contingencia. La forma más radical de contingencia se refiere a la idea misma de orden; no sólo es contingente el lugar que algo ocupa dentro de un orden establecido, sino que ese mismo orden podría ser de otra manera. La crisis de los grandes dispositivos, las totalidades de acuerdo con las cuales todas las cosas podrían ser ordenadas, impone pensar el orden y el desorden de otra manera. El descubrimiento de la complejidad nos sitúa ante un panorama en el que las cosas son cada vez menos descriptibles y la acción deviene más consciente de sus límites. Los saberes ya no constituyen un sistema de interpretación ni un sistema de acción unificante: se fragmentan, crecen en complejidad y en abstracción. Hay también una mayor conciencia de la labilidad de las construcciones y los ordenamientos, lo que se expresa en experiencias como: fragilidad, pérdida de sentido, ambigüedad, contingencia, paradojas, indeterminación, zonas de indistinción entre la regla y la excepción, entre la regla y su vulneración o entre la normalidad y el caos.

Aunque no dispongamos de denominadores comunes, principios jerárquicos o fundamentos estables que nos permitan unificar ordenadamente el mundo, sí que podemos estar seguros de que el tiempo de los ordenamientos simples ha pasado ya. Quien piense el orden de las cosas únicamente como la superación del desorden y el desorden como un fallo o una carencia, como algo esencialmente negativo, se incapacita para gestionar adecuadamente lo complejo. Las personas y las instituciones se dividen entre las que no pueden soportar el orden y las que no pueden soportar el desorden. Pero el comportamiento inteligente se mueve siempre entre esos extremos, incluso más allá de tal alternativa. Muchas experiencias no se explican mediante esa dicotomía simplificadora. No se trata ni de ignorar la distinción entre el orden y el desorden ni de hipostatizarla sino de tratarla como una distinción que hay que aprender a orquestar. Lo decisivo es pensar y actuar más allá de una simple alternativa entre el orden y el desorden, que quiere obligarnos a elegir la rigidez o la anarquía, como si entre ambos polos no hubiera un espacio para la anarquía regulada o la articulación de elementos autónomos. 
Es posible concebir el desorden como algo que permite manejarse en situaciones de elevada contingencia, en medio de constelaciones complicadas y contradictorias. En un contexto dinámico no se perdona demasiado orden, que termina por ser castigado con falta de creatividad, anquilosamiento y perplejidad. Todo orden, si quiere acreditarse como orden complejo, ha de ser enriquecido con elementos de desorden, con la fortaleza de la anarquía, con los recursos del caos (Willke 9). Son complejos precisamente aquellos sistemas que han adquirido la habilidad de poner el orden y el caos en un tipo de equilibrio especial (Waldrop, 1994, 12). Se trataría de arriesgarse a ser desordenado para articular una arquitectura del orden en los sistemas complejos, saber que un desorden en el que uno puede desenvolverse es ya una forma de orden. Hay desórdenes establecidos, que se consolidan y proporcionan alguna orientación. Siguiendo el esquema hegeliano de que la identidad resulta de articular la identidad y la no identidad (Hegel 1986, 96), Luhmann ha propuesto definir el orden como la unidad de orden y desorden; los sistemas son rutinas y azares $(2002,109)$, donde se acierta a establecer coherencias heterogéneas.

El orden implica por tanto una domesticación parcial del desorden, lo que exige una cierta tolerancia frente a la excepción. A esto se debe el hecho de que toda gestión se entienda hoy como management by exception y esa competencia se exija cada vez más. Hay una tácita vulneración de reglas que es necesaria para el pensamiento, la acción y la organización social. No toda vulneración de reglas es expresión de egoísmo de los actores o de arbitrariedad, del mismo modo que tampoco el cumplimiento de reglas implica necesariamente su correcta asimilación (está el caso de la letra muerta frente al espíritu de la ley, la forma de huelga que consiste en el cumplimiento extremado de las obligaciones laborales o la apelación a la obediencia de órdenes para liberar la propia decisión del peso de la responsabilidad; también las reglas y los procedimientos sirven para que uno se pueda disculpar). Hay una desviación de las reglas que resulta conveniente para la consecución de lo que precisamente esas reglas pretenden, del mismo modo que hay una falsificación del sentido de las reglas por parte de su aplicación literal. La vulneración forma parte del orden como los agujeros del tejido, como esa red que según la definición de Julian Barnes en El loro de Flaubert se podría describir como un conjunto de agujeros entrelazados. ¿Y si el orden no fuera otra cosa que la gestión del desorden y las reglas un conjunto de excepciones?

Todo parece indicar que el pensamiento, la acción, la sociedad no tienen otro remedio que soportar una cierta vulneración de las reglas, una transgresión de lo acostumbrado. Cabría suavizar esa paradoja si añadiéramos que esa vulneración puede tener lugar dentro de unos límites, con el fin de asegurar la flexibilidad del conjunto. En ese caso se podrían definir unos corredores para la excepción que, como suele decirse, confirmarían la regla: carnavales ocasionales que subvierten el orden y las jerarquías, que incluyen el caos en el reino del orden y de ese modo lo estabilizan, desviaciones localizadas e inofensivas, espacios de rebeldía que no inquietan en exceso al conjunto... Pero lo específico de la excepción es su resistencia frente a la funcionalización. Este tipo de estrategias no constituyen ninguna solución definitiva, ya que exigen 
regular la excepción, como si pudiera escaparse a la amenaza de que esa regulación sea a su vez vulnerada. Forma parte de la naturaleza de todo orden cultural la existencia marginal de zonas de desviación, anormalidad y discrepancia respecto de lo oficialmente regulado, y esas zonas marginales han de permanecer necesariamente borrosas.

Pero absolutizar la excepción tampoco constituye ninguna solución frente a tales imprecisiones. La extrapolación de la idea de que es imposible la repetición conduce a la hipostatización de la diferencia, lo que nos devuelve al punto de partida. Como ya advirtió Nietzsche, el valor de la excepción se malograría si fuera convertida en regla $(1980,76)$. Ni siquiera podemos consolarnos pensando, con Benjamin (1977, 697), que la excepción es la verdadera regla, pues en ese caso una ocuparía el lugar de la otra y el esquema permanecería inalterado. Convertir en regla lo excepcional implica perpetuar el problema y renunciar a pensar la articulación entre el orden y el caso particular de una manera menos rígida. Si el desorden fuera absoluto y todo excepcional, no habría propiamente excepciones, pues estas presuponen una anomalía en el orden establecido. Como muestra el caso de las falsas alarmas, una excepción convertida en norma termina por anular su carácter excepcional.

Las peculiaridades del orden ponen de manifiesto el carácter fundamentalmente heurístico del conocimiento. Si todo caso particular de una regla es siempre un caso particular, o sea un caso «especial» porque nunca se agota en su ser mero caso de una ley general, todo caso es único y tiene en sí algo de excepcional, en todo saber hay un ejercitamiento práctico que no es el entrenamiento práctico con rutinas y disposiciones específicas sino la adquisición de un juicio acerca de lo implícito. En última instancia no es sino lo que desde Aristóteles se ha Ilamado prudencia y se podría traducir como gestión de lo inesperado, capacidad de organización e improvisación, de articular lo general y particular. La tradición que va de Kant a Gadamer se refiere a esta capacidad al concebir el juicio como una actividad que tiene una dimensión de concreción, de suplemento, constructiva, creativa o genial. Se trata siempre de más que la correcta aplicación de principios generales (Gadamer 1990, 44). La cuestión del orden termina remitiendo siempre a la creatividad personal y a la inventiva de las organizaciones.

¿Cómo gestionar entonces lo inesperado? ¿Cómo prepararse para lo imprevisto? ¿Cómo ordenar lo excepcional? Pues de una manera bastante limitada, porque es propio de la vida, de su lógica flexible, que el uso de normas, órdenes y reglas esté abierto a la novedad y peculiaridad de cada situación. Por eso la integración de las organizaciones no puede asegurarse absolutamente por medio de reglamentos, diseño institucional, intenciones normativas, sino que resulta un efecto en buena medida contingente, de esa coincidencia de emergencia y auto-organización a la que aluden las modernas teorías de la complejidad. Los sistemas complejos, adaptativos, dinámicos, realizan un orden a través de la fluctuación (Prigogine), con material extremadamente inestable (Luhmann). Las dificultades del orden también representan una posibilidad: comprender mejor la fluidez del presente, reconocer de qué modo el orden y 
el desorden se entrelazan y dan lugar a nuevas formas de orden más flexibles. Un orden así no es algo que se conserva inmunizándolo frente al movimiento. La gestión del desorden no es una acción defensiva, una operación restauradora, sino una conquista, una creación constante. El orden es la continuación del caos por otros medios

\section{BIBLIOGRAFÍA}

BALANDIER Georges (1988), Le Desordre. Eloge du mouvement, Paris, Fayard.

BATESON G. (1983), Ökologie des Geistes. Anthropologische, psychologische, biologische und epistemologische Perspektiven, Frankfurt, Suhrkamp.

BOURDIEU Pierre (1987), Choses dites, Paris, Minuit.

BENJAMIN Walter (1977), Gesammelte Schriften, Frankfurt, Suhrkamp.

BORGES Jorge Luis (2003a), "El idioma analítico de John Wilkins», Otras inquisiciones, Madrid, Alianza, 154-161.

--- (2003b), «El Congreso», El libro de arena, Madrid, Alianza, 27-54.

BRAFFORT Paul (1990), «Les Bibliotheques invisibles», La Bibliothèque oulipienne, 3, Paris, Seghers, 241-266.

DERRIDA Jacques (1994), Force de loi, Paris, Galilée, 1994.

ELSTER John (1989), The cement of society, Cambridge University Press.

FOUCAULT Michel (1993), Las palabras y las cosas, México, Siglo XXI.

FREUD Sygmund (1982), "Jenseits des Lustprinzips», Studienausgabe, Frankfurt, Suhrkamp, III, 213-272.

GADAMER Hans-Georg (1990), Wahrheit und Methode. Grundzüge einer philosophischen Hermeneutik, Tübingen, Mohr.

HEGEL Georg W. F. (1986), Jenaer Schriften 1801-1807. Werke 2, Frankfurt, Suhrkamp.

KANT Immanuel (1908), Berlin, Werke (ed. Preussische Akademie der Wissenschaften).

KAULBACH Friedrich (1985), «Aspectos vigentes de la estética kantiana», en Themata (1985/2), 5-10.

KIERKEGAARD Soren (1991), Die Wiederholung, Hamburg, Rowohlt.

LUHMANN Niklas (1964), Funktionen und Folgen formaler Organisation, Berlin, Duncker \& Humboldt.

--- (1995), Soziale Systeme. Grundriss einer allgemeine Theorie, Frankfurt, Suhrkamp. 
--- (2002), Das Erziehungssystem der Gesellschaft, Frankfurt, Suhrkamp.

MORIN Edgar (1990), "Le dialogue de l'ordre et du desordre», en K. Pomian, La Querelle du déterminisme, Paris, Gallimard, 79-101.

NABOKOV Vladimir (2002), Invitado a una decapitación, Madrid, Espasa.

NIETZSCHE Friedrich (1980), Die fröhliche Wissenschaft, Kritische Studienausgabe 3, ed. Colli-Montinari, Berlin, Walter de Gruyter.

SCHÜTZ Alfred (1971), Gesammelte Aufsätze I. Studien zur phänomenologischen Philosophie, Den Haag, Nijhoff.

VALERY Paul (1960), Tel quel, Oeuvres II, Paris, La Pléiade.

VICENTE ARREGUI Jorge (1988), «El papel de la estética en la ética», en Pensamiento (1988/44), 439-453.

WALDENFELS Bernhard (1987), Ordnung im Zwielicht, Frankfurt, Suhrkamp.

WALDROP Mitchell (1994), Complexity. The emerging science at the edge of order and chaos, London, Penguin.

WILKE Helmut (2003), Heterotopia. Studien zur Krisis der Ordnung moderner Gesellschaften, Frankfurt, Suhrkamp.

WINCH Peter (1990), The idea of a social science and its relation to philosophy, London, Routledge \& Kegan Paul.

WITTGENSTEIN Ludwig (1958), The Blue and the Brown Books, Oxford, Blackwell.

--- (1984), Philosophische Untersuchungen, Werkausgabe I, Frankfurt, Suhrkamp. 